了 $31 \%$ 。过量摄入任何一种非甾体抗炎药都有可能 导致消化问题、心脏病发作和出血问题。所以, 如 果你需要服用非处方止痛药超过几天, 一定要咨询 你的医生。

\section{大量摄取维生素D或会提 高罹患骨髓疾病风险}

\section{林佑}

香港大学中医药学院

一直以来, 人们认为维生素 $\mathrm{D}$ 对人体十分有益, 维生素 D 具有抗炎、调节神经细胞功能等作用, 可 以预防佝偻病、骨质疏松等, 然而大量摄取维生素 $\mathrm{D}$ 也有可能引起骨髓病变。

最近日本神户大学医学院科研小组发现, 大量 服用维生素 D 可能是引起骨髓纤维化的原因之一。 研究结果于 2019 年 2 月发表在《血液》上刊登。

\section{骨髓病变概念}

骨髓 (bone marrow) 位于人体粗大的骨骼空腔 内, 主要由胶原蛋白细胞、造血干细胞、成纤维细胞、 血窦内皮细胞、巨噬细胞、脂肪细胞组成。

骨髓病变会造成纤细胞异常增生, 导致骨髓组 织逐渐纤维化而坏死。造血干细胞一但纤维化, 将 难以生产正常的血细胞, 导致白细胞、红细胞、血 小板生成障碍或无法发挥正常生理功能, 从而导致 众多血液疾病的出现, 例如免疫系统失调综合症、 溶血性贫血症等。

与此同时, 如果骨髓组织纤维化严重, 会令骨
参考文献

[1] https://www.msn.com/en-us/health/pain-management/thereal-difference-between-aspirin-and-ibuprofen $\% \mathrm{E} 2 \% 80 \%$ 94and-when-to-take-them/ar-BBPHPfk.

质硬化, 提高骨髓增生性血液肿瘤罹患风险, 这是 由于造血干细胞基因突变引起的。

\section{实验设计和结果}

为了了解维生素 D 会否对血液和骨骼造成影响, 研究员进行一项动物实验。实验者把 30 只小鼠平均 分成两组, 第一组 (实验组) 给予摄取大量的维生素 $\mathrm{D}$, 第二组 (对照组) 给予低剂量的维生素 D, 观察 两组小鼠身体的变化。

结果显示, 实验组小鼠骨髓干细胞受到维生素 D 强烈信号刺激, 活化免疫细胞 (泛指巨噬细胞), 这是一种病理性巨噬细胞, 它会令骨骼肌周边的成 骨细胞增生, 并诱导骨髓组织纤维化, 最终导致骨 质硬化。而对照组小鼠则没有明显身体上的变化。

而且研究人员也尝试在实验组小鼠膳食中添加 维生素 D 受体阻断剂、免疫系统抑制剂 (抑制巨噬 细胞活性), 观察其身体的变化, 结果发现能有效 预防骨髓纤维化的发生。

除此之外, 研究人员进一步解释其病理机制: 高浓度的维生素 D 之所以令干细胞生成具侵略性 的巨噬细胞, 原因在于维生素 D 令干细胞基因产 生突变, 这个异常基因已在这项小鼠实验中确定为 JAK2V61, 研究员通过与骨髓纤维化的临床病患者 进行对比, 惊讶地发现具有高度吻合的遗传学特征。

\section{参考文献}

[1] Science News. Vitamin D and immune cells stimulate bone marrow disease. February 8, 2019. 